\title{
Risk-treatment paradox in women with symptomatic coronary artery disease
}

\author{
Julinda Mehilli • Lamin King
}

Published online: 16 February 2013

(C) Springer-Verlag Berlin Heidelberg 2013

\begin{abstract}
The implementation of evidence-based treatment including diagnostic and therapeutic invasive procedures has resulted in a reduction in the mortality attributable to coronary artery disease (CAD) despite the fact that patients at high risk are less likely to have access to it. Women in particular are up to $30 \%$ less likely to receive percutaneous coronary intervention (PCI) following presentation with an acute coronary syndrome than men. This risk-treatment paradox for women is partially related to their older age, greater likelihood of comorbidities, and later presentation after symptom onset with more frequent atypical symptoms as well as to the higher proportion of symptomatic women having nonobstructive CAD. In addition, during the first decade of the PCI era, a poorer short-term survival after revascularization in women was reported while similar or better long-term outcomes than men was consistently observed. The subtly different spectrum of biological factors predisposing to high risk, frequent vascular complications, and high incidence of bleeding events, mostly related to the antithrombotic drugs over dosage, explain the early hazard seen in women. Newer antithrombotic regimens and the modern coronary stent platforms have contributed to drastic improvement of outcomes, particularly in high-risk subsets of patients including women. However, still more evidence from randomized trials with a representative proportion of women is required both to fully categorize CAD in women and to determine the most appropriate approach to therapy. Furthermore, physicians must continue to work to better identify high-risk patients
\end{abstract}

Prof. Dr. med. J. Mehilli $(\varangle) \cdot$ L. King

Medizinische Klinik und Poliklinik I, Klinikum der

Universität München, Munich, Germany

e-mail: Julinda.Mehilli@med.uni-muenchen.de and to tailor the evidence-based therapy to individual risk independent of patient's sex.

Keywords Sex · Women · Coronary intervention · Myocardial infarction $\cdot$ Stent

\section{Introduction}

Although the burden of coronary artery disease (CAD) remains enormous, the mortality attributed to it has decreased considerably during the last two decades. This is mostly derived from the implementation of evidence-based medicine including revascularization procedures [36]. The risk of the population treated determines the amount of survival benefit attributable to percutaneous coronary intervention (PCI), with ST-segment elevation myocardial infarction (STEMI) patients deriving the greatest benefit from it $[8,43]$. Paradoxical to the evidence-based research, an underuse of invasive diagnostic and therapeutic procedures has been observed in high-risk CAD patients. In particular, women, despite a frequent clinical presentation with acute coronary syndrome (ACS), are up to $30 \%$ less likely to receive cardiac catheterization than men $[8,25,43]$. Several reasons have been proposed to explain these findings.

\section{Poorer cardiovascular risk profile and frequently nonobstructive CAD in women}

Women generally develop symptomatic CAD 6-10 years later than men $[5,7,8,26,27,36]$. Because there is a significant association between age and unadjusted all-cause mortality, the oldest ACS patients derive the highest benefit from PCI compare to medical treatment alone. However, the use of 
PCI and the rate of prescription and adherence to evidencebased therapy decrease with increasing age in the CAD population [2]. The burden of comorbidity also increases with age, and this can disproportionally affect female patients. Women with CAD present more frequently with diabetes, renal insufficiency, cerebrovascular disease, and signs of congestive heart failure than men [1, 5, 7, 8, 26, 27]. Diabetes mellitus is a far more powerful coronary risk factor for women than men. It is associated with a twofold increased risk of reinfarction and a fourfold greater likelihood of developing heart failure in ACS women [19, 26, 32].

In contrast, the severity of CAD is less pronounced in women. One fifth of women presenting with ACS have angiographically documented nonobstructive CAD compared with only $10 \%$ of men $[6,14]$. These women have a fourfold increased risk of adverse nonfatal and fatal events at long term compared with asymptomatic women $[15,33]$. Impaired endothelial function of both epicardial and intramyocardial coronary arteries and differences in vascular structure may be an explanation for the presence of myocardial ischemia despite normally appearing coronary angiography in women [6].

\section{Failure to efficiently recognize ischemic symptoms and lack of awareness in women}

It is a common perception that CAD is a male disease. This is in part related to observed sex-based differences in symptoms and pain perception, with women presenting less frequently with chest pain and more often with the combination of throat, jaw, or neck discomfort and an inconclusive electrocardiogram (ECG) in comparison with men [11]. Consequently, at the time of an acute coronary event, women seek care at least $1.5 \mathrm{~h}$ later than men [12, 27]. Although the majority of studies did not report sex-specific differences in door-to-treatment time intervals [14, 27], the likelihood of getting primary reperfusion therapy is reduced in those with late presentation. Furthermore, sex-based differences in expression of cardiac biomarkers troponins and creatine kinase-MB, which is less likely elevated in women than in men [41], decrease the likelihood of women being assigned to an early PCI. Lack of awareness of warning signs may be one of the most important reasons for delayed presentation in women. Despite several national campaigns, only $8-20 \%$ of women above 35 years of age and one in five physicians were aware that cardiovascular disease was the leading cause of female deaths [34].

\section{Higher likelihood of peri-interventional complications in women}

Even after adjustment for body mass index, women have smaller coronary arteries than men [22, 26, 28, 35]. Consequent improvements in interventional techniques and devices have offset the previously observed sex-related differences in procedure-related mechanical complications [16]. However, women - mostly because of a higher occurrence of peripheral artery disease-experience peri-PCI vascular complication twice as often as men [23].

Despite receiving the same peri- and postinterventional antithrombotic treatment, women bleed more frequently than men $[11,18,23,24,30]$. Previously considered as an unavoidable and acceptable collateral damage, peri-PCI bleeding independently increases the 1-year mortality risk by a factor of three [1]. Although considerable controversy exists about the efficacy of glycoprotein IIb/IIIa inhibitors (GPI) regarding reduction of thrombotic events in women, a more pronounced relationship between GPI use and an increased incidence of bleedings among women has been consistently reported $[1,9,30]$. One of the reasons behind these findings is the 1.5-fold higher likelihood of excess dosing in women after accounting for age, body mass index, and renal function [1]. In the meantime, newer P2Y12 receptor inhibitors have substantially reduced peri-interventional thrombotic events, making the use of GPI potentially redundant $[20,31,42]$. The P2Y12 receptor is important for the potentiation of many platelet responses and for the formation of a stable hemostatic plug [13]. Different from glycoprotein IIb/IIIa receptor inhibitors-which mediate interplatelet bridging- $\mathrm{P} 2 \mathrm{Y} 12$ receptor inhibition is associated with reduction of thromboembolic events without thrombocytopenia. In the Bavarian Reperfusion Alternatives Evaluation (BRAVE) 3 trial, 800 patients with acute STEMI treated with $600 \mathrm{mg}$ of P2Y12 receptor inhibitor clopidogrel were randomly assigned to receive either GPI, abciximab, or placebo before primary PCI. Additional GPI was not associated with any additional benefit regarding infarct size and clinical events up to 1 year compared with clopidogrel alone [31,37]. No thrombocytopenia and less bleeding were observed with clopidogrel alone [31].

Furthermore, replacing unfractionated heparin plus GPI with the direct thrombin inhibitor bivalirudin has been associated with a reduction of peri-interventional risk of bleeding of up to $50 \%$ in patients with ACS [20,39]. Given that the reduction of bleeding with bivalirudin is independent of patient's sex and that women are at higher risk for bleeding independent of the drug combination used compared with men, bivalirudin could potentially be a preferred peri-interventional anticoagulant in female patients [24]. 


\section{Lower restenosis risk despite pronounced aggregation of restenosis predictors in women}

Following implantation of bare metal stents, women demonstrate more than $20 \%$ reduction of the adjusted risk for restenosis despite having a higher incidence of diabetes and smaller vessels, two major independent predictors of restenosis [28]. Use of drug-eluting stents (DES) compared with bare metal stents provides a decrease in restenosis of a similar magnitude among women and men [38]. Furthermore, the benefit derived from newer DES platforms, such as everolimus-eluting Xience stent, may be more pronounced in women than in men [35].

\section{Higher short-term but not long-term unadjusted mortality after PCI in women}

Large population-based studies have shown differences in the temporal pattern of mortality between women and men. The reported in-hospital and 30-day unadjusted mortality in women - particularly in those presenting with ACS - is up to twofold higher than in men, whereas during the course of the first year, this early excess risk gradually attenuates $[7,8,10,21,26]$. The early hazard in women seems to be less driven by sex-based differences in age and prevalence of comorbidities but mostly by the less frequent use of evidence-based diagnostic and therapeutic procedures and lifesaving medical therapy in women $[2,4,6-8,25]$. Although primary PCI has a guideline-recommended class I indication as the reperfusion strategy of choice in STEMI, it is estimated that in 2008 , less than $70 \%$ of STEMI patients underwent PCI [44]. The life-saving role of primary PCI independent from symptom onset-to-treatment time interval is well established. Women present with an ACS up to $2 \mathrm{~h}$ later than their male counterparts and would be expected to derive more benefit from primary PCI. There is a large body of evidence showing that increasing the access to evidencebased care is associated with reduced mortality for both women and men with ACS, with women deriving the greatest benefit at long term (Fig. 1) [3-5, 14, 17, 24, 26, 27, 43].

Antithrombotic therapy is essential in coronary reperfusion. It diminishes not only the risk of thrombotic epicardial vessel occlusion but also the risk of peri-PCI myocardial infarction by preventing the blockage of coronary microvasculature [27, 29]. Consequently, given the biologic differences between female and male CAD patients, with women having a more pronounced vascular and microvascular dysfunction [6], the peri-PCI GPI therapy is associated with greater amount of jeopardized myocardium salvaged in women presenting with STEMI than in men [29]. This partially explains the observed $35 \%$ reduction of age-adjusted mortality in women compared with men

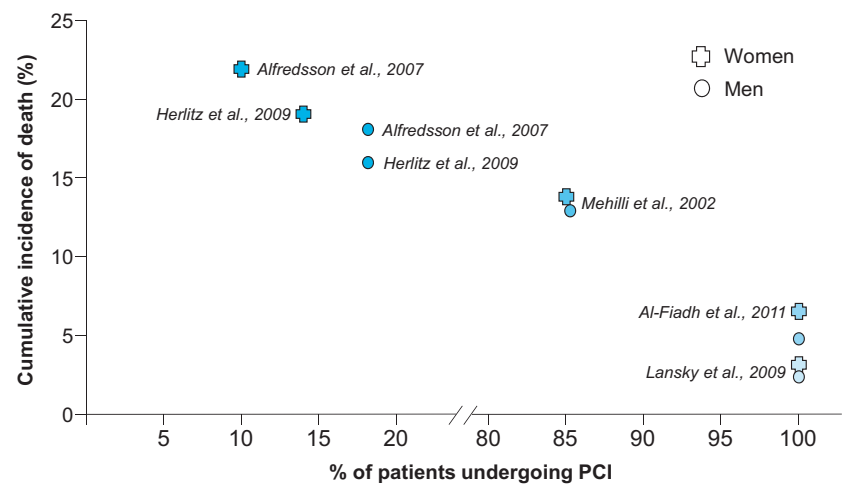

Fig. 1 One-year mortality in women and men presenting with acute coronary syndrome in relation to the proportion of patients treated with percutaneous coronary intervention (PCI)

1 year after primary PCI [27]. During the last decade, the use of GPI has been largely replaced by the newer and more potent P2Y12 receptor inhibitors. Despite the nearly 30\% increase major bleeding rates (according to Thrombolysis In Myocardial Infarction definition), prescription of prasugrel or ticagrelor for more than 1 year in patients with ACS is associated with net clinical benefit comparable to clopidogrel [40, 42]. Although female sex was the strongest independent predictor of serious bleeding associated with prasugrel in the first year following intervention in the TRITON-TIMI 38 trial, the anti-ischemic effect of newer P2Y12 receptor inhibitors was similar in women and men $[18,40,42]$. Unfortunately, we cannot draw any definite conclusion about the performance of newer therapies in women because they still remain underrepresented in randomized clinical trials. In large population study cohorts, the proportion of women presenting with ACS is as high as $40 \%$, in contrast to $25 \%$ in pivotal randomized trials $[36,39,40,42]$.

\section{Prospective}

Commonly, CAD in women is seen in the context of an additional risk factor, e.g., increased age, presence of diabetes, or comorbidities such as renal impairment and heart failure. Nevertheless, despite being at higher risk of death and demonstrating an even better response to evidencedbased therapy than men, women are underdiagnosed, undertreated, and underrepresented in randomized clinical trials. To rectify this situation, first, awareness in women of the symptoms of CAD must be increased. Second, the awareness of higher risk and poorer short-term outcomes in women must be increased among physicians and appropriate treatment tailored to the individual-specific risk must be offered. Early revascularization according to evidencebased principles, use of secondary prevention, and aggres- 
sive risk-factor control in nonobstructive disease can reduce sex-based differences in short-term mortality following ACS. Third, patient inclusion in randomized controlled trials must reflect the composition of the presenting population in terms of sex, age, and overall risk profile more accurately. This will enable a greater understanding of the subtle differences of ACS in different high-risk groups and provide a representative evidence base to determine the most appropriate treatment strategies based on individual patient risk profile.

Acknowledgments This article is part of a supplement sponsored by Lilly Deutschland GmbH and Daiichi Sankyo Deutschland GmbH.

Conflict of interest Lecture fees received from Daiichi Sankyo/Lilly, The Medicines, Abbott Vascular, Terumo, AstraZeneka.

\section{References}

1. Alexander KP, Chen AY, Newby LK et al (2006) Sex differences in major bleeding with glycoprotein IIb/IIIa inhibitors: results from the CRUSADE (Can Rapid risk stratification of Unstable angina patients Suppress ADverse outcomes with Early implementation of the ACC/AHA guidelines) initiative. Circulation 114(13):1380 1387. doi:10.1161/CIRCULATIONAHA.106.620815

2. Alexander KP, Newby LK, Armstrong PW et al (2007) Acute coronary care in the elderly, part II: ST-segment-elevation myocardial infarction: a scientific statement for healthcare professionals from the American Heart Association Council on Clinical Cardiology: in collaboration with the Society of Geriatric Cardiology. Circulation 115(19):2570-2589. doi:10.1161/ CIRCULATIONAHA.107.182616

3. Al-Fiadh AH, Andrianopoulos N, Farouque O et al (2011) Contemporary outcomes in women undergoing percutaneous coronary intervention for acute coronary syndromes. Int $\mathrm{J}$ Cardiol 151(2):195-199. doi:10.1016/j.ijcard.2010.05.018

4. Alfredsson J, Stenestrand U, Wallentin L et al (2007) Gender differences in management and outcome in non-ST-elevation acute coronary syndrome. Heart 93(11):1357-1362. doi:10.1136/ hrt.2006.102012

5. Alfredsson J, Lindback J, Wallentin L et al (2011) Similar outcome with an invasive strategy in men and women with non-ST-elevation acute coronary syndromes: from the Swedish Web-System for Enhancement and Development of Evidence-Based Care in Heart Disease Evaluated According to Recommended Therapies (SWEDEHEART). Eur Heart J 32(24):3128-3136. doi:10.1093/ eurheartj/ehr349

6. Anderson RD, Pepine CJ (2007) Gender differences in the treatment for acute myocardial infarction: bias or biology? Circulation 115(7):823-826. doi:10.1161/CIRCULATIONAHA.106.685859

7. Berger JS, Elliott L, Gallup D et al (2009) Sex differences in mortality following acute coronary syndromes. JAMA 302(8):874882. doi:10.1001/jama.2009.1227

8. Blomkalns AL, Chen AY, Hochman JS et al (2005) Gender disparities in the diagnosis and treatment of non-ST-segment elevation acute coronary syndromes: large-scale observations from the CRUSADE (Can Rapid Risk Stratification of Unstable Angina Patients Suppress Adverse Outcomes With Early Implementation of the American College of Cardiology/American Heart Association Guidelines) National Quality Improvement Initiative. J Am Coll Cardiol 45(6):832-837. doi:10.1016/j.jacc.2004.11.055
9. Boersma E, Harrington RA, Moliterno DJ et al (2002) Platelet glycoprotein IIb/IIIa inhibitors in acute coronary syndromes: a meta-analysis of all major randomised clinical trials. Lancet 359(9302):189-198

10. Daly C, Clemens F, Lopez-Sendon JL et al (2006) The impact of guideline compliant medical therapy on clinical outcome in patients with stable angina: findings from the Euro Heart Survey of stable angina. Eur Heart J 27(11):1298-1304. doi:10.1093/ eurheartj/ehl005

11. Dey S, Flather MD, Devlin G et al (2009) Sex-related differences in the presentation, treatment and outcomes among patients with acute coronary syndromes: the Global Registry of Acute Coronary Events. Heart 95(1):20-26. doi:10.1136/hrt.2007.138537

12. Diercks DB, Owen KP, Kontos MC et al (2010) Gender differences in time to presentation for myocardial infarction before and after a national women's cardiovascular awareness campaign: a temporal analysis from the Can Rapid Risk Stratification of Unstable Angina Patients Suppress ADverse Outcomes with Early Implementation (CRUSADE) and the National Cardiovascular Data Registry Acute Coronary Treatment and Intervention Outcomes Network-Get with the Guidelines (NCDR ACTION Registry-GWTG). Am Heart J 160(1):80-87 e83. doi:10.1016/j. ahj.2010.04.017

13. Dorsam RT, Kunapuli SP (2004) Central role of the P2Y12 receptor in platelet activation. The Journal of clinical investigation 113(3):340-345. doi:10.1172/JCI20986

14. Glaser R, Herrmann HC, Murphy SA et al (2002) Benefit of an early invasive management strategy in women with acute coronary syndromes. JAMA 288(24):3124-3129

15. Gulati M, Cooper-DeHoff RM, McClure C et al (2009) Adverse cardiovascular outcomes in women with nonobstructive coronary artery disease: a report from the Women's Ischemia Syndrome Evaluation Study and the St James Women Take Heart Project. Arch Intern Med 169(9):843-850. doi:10.1001/ archinternmed.2009.50

16. Hannan EL, Racz MJ, Arani DT et al (2000) A comparison of short- and long-term outcomes for balloon angioplasty and coronary stent placement. J Am Coll Cardiol 36(2):395-403

17. Herlitz J, Dellborg M, Karlsson T et al (2009) Treatment and outcome in acute myocardial infarction in a community in relation to gender. Int $\mathrm{J}$ Cardiol 135(3):315-322. doi:10.1016/j. ijcard.2008.03.065

18. Hochholzer W, Wiviott SD, Antman EM et al (2011) Predictors of bleeding and time dependence of association of bleeding with mortality: insights from the Trial to Assess Improvement in Therapeutic Outcomes by Optimizing Platelet Inhibition With Prasugrel-Thrombolysis in Myocardial Infarction 38 (TRITON-TIMI 38). Circulation 123(23):2681-2689. doi:10.1161/ CIRCULATIONAHA.110.002683

19. Kaseta JR, Skafar DF, Ram JL et al (1999) Cardiovascular disease in the diabetic woman. J Clin Endocrinol Metab 84(6):1835-1838

20. Kastrati A, Neumann FJ, Schulz S et al (2011) Abciximab and heparin versus bivalirudin for non-ST-elevation myocardial infarction. N Engl J Med 365(21):1980-1989. doi:10.1056/ NEJMoa1109596

21. Kumbhani DJ, Shishehbor MH, Willis JM et al (2012) Influence of gender on long-term mortality in patients presenting with non-ST-elevation acute coronary syndromes undergoing percutaneous coronary intervention. Am J Cardiol 109(8):1087-1091. doi:10.1016/j.amjcard.2011.11.044

22. Lansky AJ, Costa RA, Mooney M et al (2005) Gender-based outcomes after paclitaxel-eluting stent implantation in patients with coronary artery disease. J Am Coll Cardiol 45(8):1180-1185. doi:10.1016/j.jacc.2004.10.076 
23. Lansky AJ, Hochman JS, Ward PA et al (2005) Percutaneous coronary intervention and adjunctive pharmacotherapy in women: a statement for healthcare professionals from the American Heart Association. Circulation 111(7):940-953. doi:10.1161/01. CIR.0000155337.50423.C9

24. Lansky AJ, Mehran R, Cristea E et al (2009) Impact of gender and antithrombin strategy on early and late clinical outcomes in patients with non-ST-elevation acute coronary syndromes (from the ACUITY trial). Am J Cardiol 103(9):1196-1203. doi:10.1016/j.amjcard.2009.01.030

25. McAlister FA, Oreopoulos A, Norris CM et al (2007) Exploring the treatment-risk paradox in coronary disease. Arch Intern Med 167(10):1019-1025. doi:10.1001/archinte.167.10.1019

26. Mehilli J, Kastrati A, Dirschinger J et al (2000) Differences in prognostic factors and outcomes between women and men undergoing coronary artery stenting. JAMA 284(14):1799-1805

27. Mehilli J, Kastrati A, Dirschinger J et al (2002) Sex-based analysis of outcome in patients with acute myocardial infarction treated predominantly with percutaneous coronary intervention. JAMA 287(2):210-215

28. Mehilli J, Kastrati A, Bollwein H et al (2003) Gender and restenosis after coronary artery stenting. Eur Heart J 24(16):1523-1530

29. Mehilli J, Ndrepepa G, Kastrati A et al (2005) Gender and myocardial salvage after reperfusion treatment in acute myocardial infarction. J Am Coll Cardiol 45(6):828-831. doi:10.1016/j. jacc.2004.11.054

30. Mehilli J, Ndrepepa G, Kastrati A et al (2007) Sex and effect of abciximab in patients with acute coronary syndromes treated with percutaneous coronary interventions: results from Intracoronary Stenting and Antithrombotic Regimen: Rapid Early Action for Coronary Treatment 2 trial. Am Heart J 154(1):158 e151-157. doi:10.1016/j.ahj.2007.03.050

31. Mehilli J, Kastrati A, Schulz S et al (2009) Abciximab in patients with acute ST-segment-elevation myocardial infarction undergoing primary percutaneous coronary intervention after clopidogrel loading: a randomized double-blind trial. Circulation 119(14):1933-1940. doi:10.1161/ CIRCULATIONAHA.108.818617

32. Miettinen $H$, Lehto $S$, Salomaa $V$ et al (1998) Impact of diabetes on mortality after the first myocardial infarction. The FINMONICA Myocardial Infarction Register Study Group. Diabetes Care 21(1):69-75

33. Min JK, Dunning A, Lin FY et al (2011) Age- and sex-related differences in all-cause mortality risk based on coronary computed tomography angiography findings results from the International Multicenter CONFIRM (Coronary CT Angiography Evaluation for Clinical Outcomes: An International Multicenter Registry) of 23,854 patients without known coronary artery disease. J Am Coll Cardiol 58(8):849-860. doi:10.1016/j.jacc.2011.02.074
34. Mosca L, Linfante AH, Benjamin EJ et al (2005) National study of physician awareness and adherence to cardiovascular disease prevention guidelines. Circulation 111(4):499-510. doi:10.1161/01. CIR.0000154568.43333.82

35. Ng VG, Lansky AJ, Hermiller JB et al (2011) Three-year results of safety and efficacy of the everolimus-eluting coronary stent in women (from the SPIRIT III randomized clinical trial). Am J Cardiol 107(6):841-848. doi:10.1016/j.amjcard.2010.10.068

36. Roger VL, Go AS, Lloyd-Jones DM et al (2012) Heart disease and stroke statistics-2012 update: a report from the American Heart Association. Circulation 125(1):e2-e220. doi:10.1161/ CIR.0b013e31823ac046

37. Schulz S, Birkmeier KA, Ndrepepa G et al (2010) One-year clinical outcomes with abciximab in acute myocardial infarction: results of the BRAVE-3 randomized trial. Clinical research in cardiology: official journal of the German Cardiac Society 99(12):795-802. doi:10.1007/s00392-010-0185-z

38. Stefanini GG, Kalesan B, Pilgrim T et al (2012) Impact of sex on clinical and angiographic outcomes among patients undergoing revascularization with drug-eluting stents. JACC Cardiovasc Interv 5(3):301-310. doi:10.1016/j.jcin.2011.11.011

39. Stone GW, McLaurin BT, Cox DA et al (2006) Bivalirudin for patients with acute coronary syndromes. N Engl J Med 355(21):2203-2216. doi:10.1056/NEJMoa062437

40. Wallentin L, Becker RC, Budaj A et al (2009) Ticagrelor versus clopidogrel in patients with acute coronary syndromes. N Engl J Med 361(11):1045-1057. doi:10.1056/NEJMoa0904327

41. Wiviott SD, Cannon CP, Morrow DA et al (2004) Differential expression of cardiac biomarkers by gender in patients with unstable angina/non-ST-elevation myocardial infarction: a TACTICS-TIMI 18 (Treat Angina with Aggrastat and determine Cost of Therapy with an Invasive or Conservative StrategyThrombolysis In Myocardial Infarction 18) substudy. Circulation 109(5):580-586. doi:10.1161/01.CIR.0000109491.66226.26

42. Wiviott SD, Braunwald E, McCabe CH et al (2007) Prasugrel versus clopidogrel in patients with acute coronary syndromes. New Engl J Med 357(20):2001-2015. doi:10.1056/NEJMoa0706482

43. Yan AT, Yan RT, Tan M et al (2007) Management patterns in relation to risk stratification among patients with non-ST elevation acute coronary syndromes. Arch Intern Med 167(10):1009-1016. doi:10.1001/archinte.167.10.1009

44. Yeh RW, Sidney S, Chandra M et al (2010) Population trends in the incidence and outcomes of acute myocardial infarction. $\mathrm{N}$ Engl J Med 362(23):2155-2165. doi:10.1056/NEJMoa0908610 